\title{
Some characterizations of rectifying curves in the 3-dimensional hyperbolic space $\mathbb{H}^{3}(-r)$
}

\author{
Buddhadev Pal (D), Akhilesh Yadav* (iD \\ Department of Mathematics, Institute of Science, Banaras Hindu University, Varanasi-221005, India
}

\begin{abstract}
In this paper, we study the geometry of rectifying curves in the 3-dimensional hyperbolic space $\mathbb{H}^{3}(-r)$. Further we obtain the distance function in terms of arc length when the rectifying curve lying in the upper half plane. Then we find the distance function and also give the general equations of the curvature and torsion of rectifying general helices in $\mathbb{H}^{3}(-r)$.
\end{abstract}

Mathematics Subject Classification (2020). 53C50, 53C40, 53A04, 53A05

Keywords. rectifying curve, general helix, geodesic, hyperbolic space $\mathbb{H}^{3}(-r)$

\section{Introduction}

In [4], B.Y. Chen gave the idea that the ratio of torsion and curvature of a regular curve is a linear function of arc length $s$, i.e., $(\tau / \kappa)(s)=c_{1} s+c_{2}$ for some constants $c_{1}$ and $c_{2}$. If $c_{1}=0$, we obtain generalized helices; otherwise, we obtain rectifying curves. A space curve whose position vector always lies in its rectifying plane is called rectifying curve. So, a curve $\gamma$ is said to be rectifying curve if there exist a point $r$ in $\mathbb{R}^{3}$ such that $\gamma(s)-r=C_{1} B(s)+C_{2} T(s)$, where $C_{1}, C_{2}$ are some function of arc length $s$. Now the Frenet frame: $T=\gamma^{\prime}, N, B=T \times N$ of a unit speed curve $\gamma$ in $\mathbb{R}^{3}$ satisfies the Serret-Frenet equations:

$$
\left(\begin{array}{l}
T^{\prime} \\
N^{\prime} \\
B^{\prime}
\end{array}\right)=\left(\begin{array}{ccc}
0 & \kappa & 0 \\
-\kappa & 0 & \tau \\
0 & -\tau & 0
\end{array}\right)\left(\begin{array}{l}
T \\
N \\
B
\end{array}\right),
$$

where the function $\kappa(s)>0$ and $\tau(s)$ are called the curvature and the torsion of the curve and the above matrix is skew-symmetric. Therefore at each point of the curve we always get three planes namely: $\{\mathrm{T}, \mathrm{N}\}$-osculating plane, $\{\mathrm{N}, \mathrm{B}\}$-normal plane, $\{\mathrm{B}, \mathrm{T}\}$-rectifying plane and the equations of the corresponding planes are $(R-r) \cdot B=0,(R-r) \cdot T=0,(R-r) \cdot N=$ 0 , where $R$ - position vector of any point on the respective plane, $r$-position vector of a specified point of the given curve. To know more about the characterization of rectifying curve we refer the reader to see $[1,2,6]$. In [7], P. Lucas and J.A.O. Yagues, studied rectifying curves in the three-dimensional hyperbolic space, and obtain some results of characterization and classification for such kind of curves.

\footnotetext{
${ }^{*}$ Corresponding Author.

Email addresses: pal.buddha@gmail.com (B. Pal), akhilesh_mathau@rediffmail.com (A. Yadav)

Received: 16.04.2019; Accepted: 01.06.2020
} 
In [5], S. Izumiya and N. Takeuchi introduced the notion of slant helix, if the principle normal lines of $\gamma$ makes a constant angle with a fixed direction, also they found a necessary and sufficient condition for a curve $\gamma$ with $\kappa(s)>0$ to be a slant helix is that function $\sigma=\frac{\kappa^{2}}{\left(\kappa^{2}+\tau^{2}\right)^{3 / 2}}\left(\frac{\tau}{\kappa}\right)^{\prime}$ be constant. Further in [8], P. Lucas and J.A.O Yagues studied slant helices in the three dimensional sphere. Also in [3], M. Barros gave the definition of Lancret curve (general helix), the principle normal lines are perpendicular to a fixed direction. Thus a general helix is the special case of a slant helix. It is clear that if $\sigma \equiv 0$ then $\gamma$ is a general helix. Also M. Barros gave a theorem that, a curve $\gamma$ in $\mathbb{H}^{3}$ is a slant helix if and only if either $\gamma$ is a curve in some unit hyperbolic plane $\mathbb{H}^{2} \subset \mathbb{H}^{3}$ with $\tau \equiv 0$ or $\gamma$ is a helix in $\mathbb{H}^{3}$.

Thus motivated sufficiently we study general helices in the 3-dimensional hyperbolic space $\mathbb{H}^{3}(-r)$ and obtain several results corresponding to the rectifying general helix and characterization of rectifying curve in $\mathbb{H}^{3}(-r)$. Our work is organized as follows: using the Gauss formula and the definition of rectifying curve in $\mathbb{H}^{3}(-r)$, we find expressions of $T^{0^{\prime}}{ }_{\gamma}, N^{0^{\prime}}{ }_{\gamma}, B^{0^{\prime}}{ }_{\gamma}, T^{0^{\prime}}{ }_{\phi_{s}} \cdot T^{0^{\prime}} \bar{\gamma}, N^{0^{\prime}}{ }_{\phi_{s}} \cdot N^{0^{\prime}} \bar{\gamma}, B^{0^{\prime}}{ }_{\phi_{s}} \cdot B^{0^{0^{\prime}}} \bar{\gamma}$ etc. Here we take dot product because it gives the geometrical interpretation of curve. Further we obtain the distance function in $\mathbb{H}^{3}(-r)$ in terms of $\lambda$ and $\mu$, which satisfy some differential equation. We also find distance function in terms of arc length when the rectifying curve lying in the upper half plane. Next we find some characterizations of rectifying curve in $\mathbb{H}^{3}(-r)$. Finally we give the general equations of the curvature and torsion of a rectifying general helix.

\section{Preliminaries}

Let $\mathbb{H}^{3}(p,-r)=\left\{x=\left(x_{1}, x_{2}, x_{3}, x_{4}\right) \in \mathbb{R}_{1}^{4} \mid\left\langle x-p, x-p>=-r^{2}, x_{1}>0\right\} \subset \mathbb{R}_{1}^{4}\right.$ be the hyperbolic space with centered at $p \in \mathbb{R}_{1}^{4}$ and radius $r>0$, where $\mathbb{R}_{1}^{4}$ is the four dimensional Lorentzian manifold with flat metric $g=-d x_{1}^{2}+d x_{2}^{2}+d x_{3}^{2}+d x_{4}^{2}$. Also we denote $\mathbb{H}^{3}(0,-r) \equiv \mathbb{H}^{3}(-r)=\left\{x \in \mathbb{R}_{1}^{4} \mid-x_{1}^{2}+x_{2}^{2}+x_{3}^{2}+x_{4}^{2}=-r^{2}, x_{1}>0\right\} \subset \mathbb{R}_{1}^{4}$ and $\mathbb{H}^{3}(0,-1) \equiv \mathbb{H}^{3}$.

We know that if $\bar{\nabla}$ and $\nabla^{\circ}$ denote the Levi-Civita connections on $\mathbb{H}^{3}(-r)$ and $\mathbb{R}_{1}^{4}$ respectively then they are related by the Gauss formula, $\nabla_{X}^{\circ} Y=\bar{\nabla}_{X} Y+\frac{1}{r^{2}}<X, Y>\phi$, where $\phi: \mathbb{H}^{3}(-r) \rightarrow \mathbb{R}_{1}^{4}$ denotes the position vector and $X, Y$ are vector fields tangent to $\mathbb{H}^{3}(-r)$. Let us consider a unit speed curve $\gamma: I \subset \mathbb{R} \rightarrow \mathbb{H}^{3}(-r)$ and assume that $\gamma$ is not a geodesic curve then we always get $\nabla_{T_{\gamma}}^{\circ} T_{\gamma}=\kappa_{\gamma} N_{\gamma}+\frac{1}{r^{2}} \gamma, \nabla_{T_{\gamma}}^{\circ} N_{\gamma}=-\kappa_{\gamma} T_{\gamma}+\tau_{\gamma} B_{\gamma}, \nabla_{T_{\gamma}}^{\circ} B_{\gamma}=$ $-\tau_{\gamma} N_{\gamma}$, where two functions $\kappa_{\gamma}>0$ and $\tau_{\gamma}$ are curvature and torsion of the curve $\gamma$. It is also well-known that the principle normal geodesic in $\mathbb{H}^{3}(-r)$ starting at $\gamma(s)$ of the curve $\gamma$ can be defined as the geodesic curve parameterized by $\phi_{s}(t)=\exp _{\gamma(s)}\left(t N_{\gamma}(s)\right)=$ $\cosh \left(\frac{t}{r}\right) \gamma(s)+r \sinh \left(\frac{t}{r}\right) N_{\gamma}(s), t \in \mathbb{R}$.

In [7], authors gave two equivalent definitions of rectifying curve in the three dimensional hyperbolic space.

Definition 2.1. A unit speed curve $\gamma=\gamma(s)(s \in I)$ in $\mathbb{H}^{3}(-r)$, with $\kappa_{\gamma}>0$, is said to be rectifying curve if there exists a point $p \in \mathbb{H}^{3}(-r)$ such that $p$ is not belongs to $\operatorname{Im}(\gamma) \equiv \gamma(I)$ and the geodesics connecting $p$ with $\gamma(s)$ are orthogonal to the principle normal geodesics at $\gamma(s)$, for all $s$.

Definition 2.2. The geodesics connecting $p$ with $\gamma(s)$ are tangent to the rectifying plane of $\gamma$ i.e., the planes generated by $\left\{T_{\gamma}(s), B_{\gamma}(s)\right\}$.

Also in [7], two characterization theorems for rectifying curves are given.

Theorem 2.3. Let $\gamma=\gamma(s)(s \in I)$ be a unit speed curve in $\mathbb{H}^{3}(-r)$. Then, $\gamma$ is a rectifying curve if and only if the ratio of torsion and curvature of the curve is given by $\frac{\tau_{\gamma}}{\kappa_{\gamma}}(s)=c_{1} \sinh \left(\frac{s+s_{0}}{r}\right)+c_{2} \cosh \left(\frac{s+s_{0}}{r}\right)$, for some constants $c_{1}, c_{2}$ and $s_{0}$, with $1-c_{1}{ }^{2}+c_{2}{ }^{2}<$ 0 . 
Theorem 2.4. Let $p \in H^{3}(-r)$ and consider a unit speed curve $V(t)$ in $S^{2}(1) \subset T_{p} H^{3}(-r)$. Then, for any nonzero function $\rho(t)$, the curvature $\kappa_{\gamma}$ and the speed $v$ of the curve $\gamma(t)=\exp _{p}(\rho(t) V(t))$, and the geodesic curvature $\kappa_{V}$ of $V$ satisfy the inequality $\kappa_{V}^{2} \leq$ $\frac{v^{4} \kappa_{\gamma}^{2}}{r^{2} \sinh ^{2}(\rho / r)}$, with the equality sign holding identically if and only if $\gamma$ is a rectifying

\section{Main results}

Theorem 3.1. Let $\gamma: I \subset \mathbb{R} \rightarrow \mathbb{H}^{3}(-r)$ be a unit speed rectifying curve in $\mathbb{H}^{3}(-r)$. If $\left\{T_{\gamma}, N_{\gamma}, B_{\gamma}\right\}$ is the Frenet frame along $\gamma$ and $\bar{\nabla}$ and $\nabla^{\circ}$ denote the Levi-Civita connections on $\mathbb{H}^{3}(-r)$ and $\mathbb{R}_{1}^{4}$ respectively then by using the Gauss formula the Frenet equations of $\gamma$ can be written as follows:

$$
T_{\gamma}^{\mathrm{o}^{\prime}}=\kappa_{\gamma} N_{\gamma}+1 / r^{2} \gamma, N_{\gamma}^{\mathrm{o}^{\prime}}=-\kappa_{\gamma} T_{\gamma}+\kappa_{\gamma} \psi B_{\gamma}, B_{\gamma}^{\mathrm{o}^{\prime}}=-\kappa_{\gamma} \psi N_{\gamma},
$$

where $\kappa_{\gamma}, \tau_{\gamma}$ denote the curvature and torsion of $\gamma$, which satisfy any of the following conditions:

(1) $T^{\circ^{\prime}} \phi_{s} \cdot T^{0^{\prime}} \bar{\gamma}=\frac{1}{r^{2}}\left(\kappa_{\phi_{s}} N_{\phi_{s}} \cdot \bar{\gamma}+\kappa_{\bar{\gamma}} \phi_{s} \cdot N_{\bar{\gamma}}+\frac{1}{r^{2}} \phi_{s} \cdot \bar{\gamma}\right)$,

$N^{\mathrm{o}^{\prime}}{ }_{\phi_{s}} \cdot N^{\mathrm{o}^{\prime}} \bar{\gamma}=\lambda_{1} \tau_{\phi_{s}} \tau_{\bar{\gamma}}$,

$B^{\mathrm{o}^{\prime}} \phi_{s} \cdot{B^{\circ}}_{\bar{\gamma}}=0$.

(2) $T_{\phi_{s}}^{\circ^{\prime}} \cdot T^{\mathrm{o}^{\prime}}{ }_{\bar{\gamma}}=\lambda_{4} \kappa_{\phi_{s}} \kappa_{\bar{\gamma}}+\frac{1}{r^{2}}\left(\lambda_{4} \kappa_{\phi_{s}} \bar{\gamma}+\phi_{s} \kappa_{\bar{\gamma}}\right) \cdot N_{\bar{\gamma}}+\frac{1}{r^{4}} \phi_{s} \cdot \bar{\gamma}, N_{\phi_{s}}^{\mathrm{o}^{\prime}} \cdot N^{\mathrm{o}^{\prime}} \bar{\gamma}=-\lambda_{2} \tau_{\phi_{s}} \kappa_{\bar{\gamma}}-$ $\lambda_{3} \kappa_{\phi_{s}} \tau_{\bar{\gamma}}, B^{\mathrm{o}^{\prime}}{ }_{\phi_{s}} \cdot B^{\mathrm{o}^{\prime}} \bar{\gamma}=-\lambda_{4} \tau_{\phi_{s}} \tau_{\bar{\gamma}}$.

(3) $T^{\mathrm{o}^{\prime}}{ }_{\phi_{s}} \cdot T^{\mathrm{o}^{\prime}} \bar{\gamma}=\frac{1}{r^{2}}\left(\kappa_{\phi_{s}} N_{\phi_{s}} \cdot \bar{\gamma}+\kappa_{\bar{\gamma}} \phi_{s} \cdot N_{\bar{\gamma}}+\frac{1}{r^{2}} \phi_{s} \cdot \bar{\gamma}\right), N^{\mathrm{o}^{\prime}}{ }_{\phi_{s}} \cdot N^{\mathrm{o}^{\prime}} \bar{\gamma}=-d_{1} \tau_{\phi_{s}} \kappa_{\bar{\gamma}}$, $B^{\mathrm{o}^{\prime}}{ }_{\phi_{s}(t)} \cdot B^{\mathrm{o}^{\prime}} \bar{\gamma}=0$.

(4) $T^{\mathrm{o}^{\prime}}{ }_{\phi_{s}} \cdot T^{\mathrm{o}^{\prime}} \bar{\gamma}=\frac{1}{r^{2}}\left(\kappa_{\phi_{s}} N_{\phi_{s}} \cdot \bar{\gamma}+\kappa_{\bar{\gamma}} \phi_{s} \cdot N_{\bar{\gamma}}+\frac{1}{r^{2}} \phi_{s} \cdot \bar{\gamma}\right), N^{\mathrm{o}^{\prime}}{ }_{\phi_{s}} \cdot N^{\mathrm{o}^{\prime}} \bar{\gamma}=-d_{2} \kappa_{\phi_{s}} \tau_{\bar{\gamma}}$, $B^{\mathrm{o}^{\prime}}{ }_{\phi_{s}(t)} \cdot B^{\mathrm{o}^{\prime}} \bar{\gamma}=0$

where $\lambda_{1}, \lambda_{2}, \lambda_{3}, \lambda_{4}, d_{1}, d_{2} \in \mathbb{R}$.

Proof. Let $\gamma: I \subset \mathbb{R} \rightarrow \mathbb{H}^{3}(-r)$ be a unit speed rectifying curve in $\mathbb{H}^{3}(-r)$. If $\left\{T_{\gamma}, N_{\gamma}, B_{\gamma}\right\}$ be the Frenet frame along $\gamma$ and $\bar{\nabla}$ and $\nabla^{\circ}$ denote the Levi-Civita connections on $\mathbb{H}^{3}(-r)$ and $\mathbb{R}_{1}^{4}$ respectively then the Frenet equations of $\gamma$ are

$$
\bar{\nabla}_{T_{\gamma}} T_{\gamma}=\kappa_{\gamma} N_{\gamma}, \bar{\nabla}_{T_{\gamma}} N_{\gamma}=-\kappa_{\gamma} T_{\gamma}+\tau_{\gamma} B_{\gamma}, \bar{\nabla}_{T_{\gamma}} B_{\gamma}=-\tau_{\gamma} N_{\gamma}
$$

where functions $\kappa_{\gamma}>0$ and $\tau_{\gamma}$ are curvature and torsion of the curve $\gamma$. After using the Gauss formula in (3.1), we get

$$
\nabla_{T_{\gamma}}^{\circ} T_{\gamma}=\kappa_{\gamma} N_{\gamma}+\frac{1}{r^{2}} \gamma, \nabla_{T_{\gamma}}^{\circ} N_{\gamma}=-\kappa_{\gamma} T_{\gamma}+\tau_{\gamma} B_{\gamma}, \nabla_{T_{\gamma}}^{\circ} B_{\gamma}=-\tau_{\gamma} N_{\gamma} .
$$

Then from ([7], Theorem 3.), using the relation of $\tau_{\gamma}$ and $\kappa_{\gamma}$ for rectifying curve we obtain,

$$
\nabla^{\circ} T_{\gamma} T_{\gamma}=\kappa_{\gamma} N_{\gamma}+\frac{1}{r^{2}} \gamma, \nabla^{\circ}{ }_{T_{\gamma}} N_{\gamma}=-\kappa_{\gamma} T_{\gamma}+\kappa_{\gamma} \psi B_{\gamma}, \nabla^{\circ}{ }_{T_{\gamma}} B_{\gamma}=-\kappa_{\gamma} \psi N_{\gamma},
$$

where $\psi(s)=c_{1} f(s)+c_{2} g(s)$. Now, we write the equation (3.3) in the following notation

$$
T_{\gamma}^{\mathrm{o}^{\prime}}=\kappa_{\gamma} N_{\gamma}+\frac{1}{r^{2}} \gamma, N_{\gamma}^{\mathrm{o}^{\prime}}=-\kappa_{\gamma} T_{\gamma}+\kappa_{\gamma} \psi B_{\gamma}, B^{\mathrm{o}^{\prime}}{ }_{\gamma}=-\kappa_{\gamma} \psi N_{\gamma} .
$$

Now, using Definition 2.1, let $\phi_{s}(t)$ be geodesics connecting $p$ with $\gamma(s)$ are orthogonal to the principle normal geodesics $\bar{\gamma}$ at $\gamma(s)$, for all $s$. Then we get,

$$
\begin{aligned}
& T_{\phi_{s}(t)}^{\mathrm{o}^{\prime}}=\kappa_{\phi_{s}(t)} N_{\phi_{s}(t)}+\frac{1}{r^{2}} \phi_{s}(t), \\
& N^{\mathrm{o}^{\prime}}{ }_{\phi_{s}(t)}=-\kappa_{\phi_{s}(t)} T_{\phi_{s}(t)}+\tau_{\phi_{s}(t)} B_{\phi_{s}(t)} \text {, } \\
& B^{\mathrm{o}^{\prime}}{ }_{\phi_{s}(t)}=-\tau_{\phi_{s}(t)} N_{\phi_{s}(t)} \text {, }
\end{aligned}
$$


and

$$
\begin{aligned}
& T^{\mathrm{o}^{\prime}} \bar{\gamma}=\kappa_{\bar{\gamma}} N_{\bar{\gamma}}+\frac{1}{r^{2}} \bar{\gamma}, \\
& {N^{\mathrm{o}^{\prime}}}_{\bar{\gamma}}=-\kappa_{\bar{\gamma}} T_{\bar{\gamma}}+\tau_{\bar{\gamma}} B_{\bar{\gamma}}, \\
& {B^{\mathrm{o}^{\prime}}}_{\bar{\gamma}}=-\tau_{\bar{\gamma}} N_{\bar{\gamma}} .
\end{aligned}
$$

Now for the case of rectifying curve, $\phi_{s}(t)$ and $\bar{\gamma}(s)$ are orthogonal at $\gamma(s)$ for all $s$ i.e., $T_{\phi_{s}(t)} \cdot T_{\bar{\gamma}}=0$ and we get two cases corresponding to the Frenet frame of the curves $\phi_{s}$ and $\bar{\gamma}$.

\section{Case 1.}

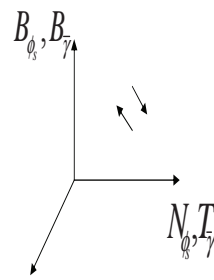

$T_{\phi_{s}}, N_{\bar{\gamma}}$

Condition (i)

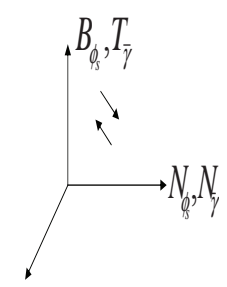

$T_{\phi_{s}}, B_{\bar{\gamma}}$

Condition (ii)

Then using Condition (i) in the equations (3.5) and (3.6), we get

$$
\begin{gathered}
T_{\phi_{s}}^{\mathrm{o}^{\prime}} \cdot T^{\mathrm{o}^{\prime}} \bar{\gamma}=\frac{1}{r^{2}}\left(\kappa_{\phi_{s}} N_{\phi_{s}} \cdot \bar{\gamma}+\kappa_{\bar{\gamma}} \phi_{s} \cdot N_{\bar{\gamma}}+\frac{1}{r^{2}} \phi_{s} \cdot \bar{\gamma}\right), \\
N_{\phi_{s}}^{\mathrm{o}^{\prime}} \cdot N^{\mathrm{o}^{\prime}}{ }_{\bar{\gamma}}=\lambda_{1} \tau_{\phi_{s}} \tau_{\bar{\gamma}} B_{\bar{\gamma}} \cdot B_{\bar{\gamma}}=\lambda_{1} \tau_{\phi_{s}} \tau_{\bar{\gamma}},{B^{\mathrm{o}^{\prime}}}_{\phi_{s}(t)} \cdot B^{\mathrm{o}^{\prime}} \bar{\gamma}=0,
\end{gathered}
$$

where $B_{\phi_{s}}=\lambda_{1} B_{\bar{\gamma}}$. By using Condition (ii) in the equations (3.5) and (3.6), we obtain

$$
\begin{aligned}
& T_{\phi_{s}}^{\mathrm{o}^{\prime}} \cdot T^{\mathrm{o}^{\prime}}{ }_{\bar{\gamma}}=\kappa_{\phi_{s}} \kappa_{\bar{\gamma}} N_{\phi_{s}} \cdot N_{\bar{\gamma}}+\frac{1}{r^{2}}\left(\kappa_{\phi_{s}} N_{\phi_{s}} \cdot \bar{\gamma}+\kappa_{\bar{\gamma}} \phi_{s} \cdot N_{\bar{\gamma}}+\frac{1}{r^{2}} \phi_{s} \cdot \bar{\gamma}\right) \\
& =\lambda_{4} \kappa_{\phi_{s}} \kappa_{\bar{\gamma}}+\frac{1}{r^{2}}\left(\lambda_{4} \kappa_{\phi_{s}} \bar{\gamma}+\kappa_{\bar{\gamma}} \phi_{s}\right) \cdot N_{\bar{\gamma}}+\frac{1}{r^{4}} \phi_{s} \cdot \bar{\gamma}, \\
& N^{\mathrm{o}^{\prime}}{ }_{\phi_{s}} \cdot N^{\mathrm{o}^{\prime}}{ }_{\bar{\gamma}}=-\lambda_{2} \tau_{\phi_{s}} \kappa_{\bar{\gamma}} T_{\bar{\gamma}} \cdot T_{\bar{\gamma}}-\lambda_{3} \kappa_{\phi_{s}} \tau_{\bar{\gamma}} B_{\bar{\gamma}} \cdot B_{\bar{\gamma}}=-\lambda_{2} \tau_{\phi_{s}} \kappa_{\bar{\gamma}}-\lambda_{3} \kappa_{\phi_{s}} \tau_{\bar{\gamma}} \text {, } \\
& B^{\mathrm{o}^{\prime}}{ }_{\phi_{s}} \cdot B^{\mathrm{o}^{\prime}}{ }_{\bar{\gamma}}=\lambda_{4} \tau_{\phi_{s}} \tau_{\bar{\gamma}},
\end{aligned}
$$

where $B_{\phi_{s}}=\lambda_{2} T_{\bar{\gamma}}, T_{\phi_{s}}=\lambda_{3} B_{\bar{\gamma}}$ and $N_{\phi_{s}}=\lambda_{4} N_{\bar{\gamma}}$. Now we know that $T_{\gamma}$ can be written as $T_{\gamma}=c_{1} T_{\bar{\gamma}}+c_{2} N_{\bar{\gamma}}+c_{3} B_{\bar{\gamma}}$, and $T_{\gamma}=c^{\prime}{ }_{1} T_{\phi_{s}}+c^{\prime}{ }_{2} N_{\phi_{s}}+c^{\prime}{ }_{3} B_{\phi_{s}}$. Also we know that $T_{\gamma} \cdot T_{\gamma}=1$, therefore after using Condition (ii), we get

$$
\begin{gathered}
c_{1} c^{\prime}{ }_{3} T_{\bar{\gamma}} \cdot B_{\phi_{s}}+c_{2} c^{\prime}{ }_{2} N_{\bar{\gamma} \cdot} \cdot N_{\phi_{s}}+c_{3} c^{\prime}{ }_{1} B_{\bar{\gamma}} \cdot T_{\phi_{s}}=1, \\
\Rightarrow c_{1} c^{\prime}{ }_{3} \lambda_{2}+c_{2} c_{2}{ }_{2} \lambda_{4}+c_{3} c^{\prime}{ }_{1} \lambda_{3}=1 . \\
\Rightarrow c_{1} c^{\prime}{ }_{3} \lambda_{2}+c_{3} c^{\prime}{ }_{1} \lambda_{3}=1-c_{2} c_{2}{ }_{2} d_{3},
\end{gathered}
$$

where we consider $\lambda_{4}=d_{3} \in \mathbb{R}$. Thus we get

$$
c \lambda_{2}+d \lambda_{3}=n
$$

where $c=c_{1} c^{\prime}{ }_{3}, d=c_{3} c^{\prime}{ }_{1}, n=1-c_{2} c_{2}{ }_{2} d_{3}$. 
On the other hand we can write $N_{\bar{\gamma}}=b_{1} T_{\phi_{s}}+b_{2} N_{\phi_{s}}+b_{3} B_{\phi_{s}}$ and $N_{\phi_{s}}=b_{1}^{\prime} T_{\bar{\gamma}}+b_{2}^{\prime} N_{\bar{\gamma}}+$ $b_{3}^{\prime} B_{\bar{\gamma}}$. Now, taking the dot product of $N_{\bar{\gamma}}$ and $N_{\phi_{s}}$, and then using Condition (ii), we get $b_{3} b_{1}^{\prime} \lambda_{2}+b_{1} b_{3}^{\prime} \lambda_{3}=\left(1-b_{2} b_{2}^{\prime}\right) d_{3}=m$, which implies

$$
a \lambda_{2}+b \lambda_{3}=m
$$

where $a=b_{3} b_{1}^{\prime}, b=b_{1} b_{3}^{\prime}, m=\left(1-b_{2} b_{2}^{\prime}\right) d_{3}$ and $c_{1}, c_{2}, c_{3}, c_{1}^{\prime}, c_{2}^{\prime}, c_{3}^{\prime}, b_{1}, b_{2}, b_{3}, b_{1}^{\prime}, b_{2}^{\prime}, b_{3}^{\prime}$, $a, b, c, d, m, n, \lambda_{1}, \lambda_{2}, \lambda_{3}, \lambda_{4} \in \mathbb{R}$.

On solving the equations (3.7) and (3.8), we get $\lambda_{2}=\frac{d m-b n}{a d-c b}, \lambda_{3}=\frac{c m-a n}{c b-a d}$. Similarly, using Condition (i), $\lambda_{1}$ can also be calculated.

\section{Case 2.}

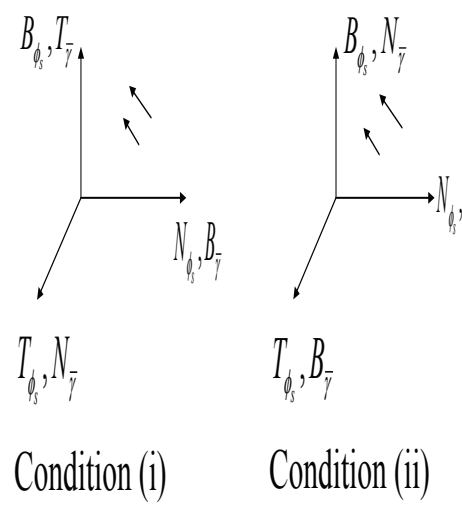

Then using Condition (i) in the equations (3.5) and (3.6), we get

$$
\begin{gathered}
T_{{ }_{\phi_{s}}{ }^{\prime}} \cdot T^{\mathrm{o}^{\prime}}{ }_{\bar{\gamma}}=\frac{1}{r^{2}}\left(\kappa_{\phi_{s}} N_{\phi_{s}} \cdot \bar{\gamma}+\kappa_{\bar{\gamma}} \phi_{s} \cdot N_{\bar{\gamma}}+\frac{1}{r^{2}} \phi_{s} \cdot \bar{\gamma}\right), \\
N^{\mathrm{o}^{\prime}}{ }_{\phi_{s}} \cdot N^{\mathrm{o}^{\prime}}{ }_{\bar{\gamma}}=-\tau_{\phi_{s}} \kappa_{\bar{\gamma}} T_{\bar{\gamma}} \cdot B_{\phi_{s}}=-d_{1} \tau_{\phi_{s}} \kappa_{\bar{\gamma}}, B^{\mathrm{o}^{\prime}}{ }_{\phi_{s}(t)} \cdot B^{\mathrm{o}^{\prime}}{ }_{\bar{\gamma}}=0,
\end{gathered}
$$

where $B_{\phi_{s}}=d_{1} T_{\bar{\gamma}}$. By using Condition (ii) in the equations (3.5) and (3.6), we get

$$
\begin{gathered}
T_{{ }_{\phi_{s}}{ }^{\prime}} \cdot T^{\mathrm{o}^{\prime}}{ }_{\bar{\gamma}}=\frac{1}{r^{2}}\left(\kappa_{\phi_{s}} N_{\phi_{s}} \cdot \bar{\gamma}+\kappa_{\bar{\gamma}} \phi_{s} \cdot N_{\bar{\gamma}}+\frac{1}{r^{2}} \phi_{s} \cdot \bar{\gamma}\right), \\
N^{\mathrm{o}^{\prime}}{ }_{\phi_{s}} \cdot N^{\mathrm{o}^{\prime}}{ }_{\bar{\gamma}}=-\kappa_{\phi_{s}} \tau_{\bar{\gamma}} T_{\phi_{s}} \cdot B_{\bar{\gamma}}=-d_{2} \kappa_{\phi_{s}} \tau_{\bar{\gamma}}, B^{\mathrm{o}^{\prime}}{ }_{\phi_{s}(t)} \cdot{B^{\circ}}^{\mathrm{o}^{\prime}}=0,
\end{gathered}
$$

where $T_{\phi_{s}}=d_{2} B_{\bar{\gamma}}$. Then from above procedure we can find the values of $d_{1}, d_{2} \in \mathbb{R}$. Thus, we obtain the required results.

Theorem 3.2. Let $\gamma=\gamma(s)$ be a unit speed rectifying curve in $\mathbb{H}^{3}(-r)$. Then the distance function $\rho=\|\gamma\|$ satisfies $\rho^{2}=-\lambda^{2}+\mu^{2}$, where $\lambda$ and $\mu$ satisfy the equation $\left(1-\lambda^{\prime}\right) a T_{\bar{\gamma}}-$ $\left(b-b \lambda^{\prime}+\mu^{\prime}\right) B_{\gamma}+\frac{\lambda \gamma}{r^{2}}=\lambda T_{\gamma}^{\circ^{\prime}}+\mu B_{\gamma}^{\circ^{\prime}}$ and $a, b \in \mathbb{R}$.

Proof. Let $\gamma=\gamma(s)$ be a unit speed rectifying curve in $\mathbb{H}^{3}(-r)$. Then position vector $\gamma$ of a curve satisfies the equation

$$
\gamma(s)=\lambda(s) T_{\gamma}(s)+\mu(s) B_{\gamma}(s),
$$

where $\lambda(s)$ and $\mu(s)$ are differential functions. Now, differentiating the equation (3.9) with respect to $s$ and using Frenet equations, we get $T_{\gamma}(s)=\lambda^{\prime}(s) T_{\gamma}(s)+\lambda(s)\left(T_{\gamma}^{o^{\prime}}-\frac{1}{r^{2}} \gamma\right)+$ $\mu^{\prime}(s) B_{\gamma}(s)+\mu B_{\gamma}^{\circ}$, which implies

$$
\left(1-\lambda^{\prime}\right) T_{\gamma}-\mu^{\prime} B_{\gamma}-\lambda T_{\gamma}^{\mathrm{o}^{\prime}}-\mu B_{\gamma}^{\mathrm{o}^{\prime}}+\frac{\lambda \gamma}{r^{2}}=0 .
$$


Then using Definition 2.1 of rectifying curve in $\mathbb{H}^{3}(-r), T_{\gamma}$ can be written in the form, $T_{\gamma}=a T_{\bar{\gamma}}-b B_{\gamma}$, where $\bar{\gamma}$ is the geodesics connecting $p$ with $\gamma(s)$ are tangent to the rectifying plane of $\gamma$ i.e., the planes generated by $\left\{T_{\gamma}(s), B_{\gamma}(s)\right\}$. Therefore the equation (3.10) can be rewritten as

$$
\left(1-\lambda^{\prime}\right) a T_{\bar{\gamma}}-\left(b-b \lambda^{\prime}+\mu^{\prime}\right) B_{\gamma}+\frac{\lambda \gamma}{r^{2}}=\lambda T_{\gamma}^{0^{\prime}}+\mu B_{\gamma}^{o^{\prime}} .
$$

Also from the equation (3.9), it is clear that the distance function $\rho^{2}=\|\gamma\|^{2}=|g(\gamma, \gamma)|=$ $-\lambda^{2}+\mu^{2}$, where $\lambda$ and $\mu$ satisfy the equation (3.11). Thus the proof is completed.

Theorem 3.3. Let $\gamma=\gamma(s)$ be a unit speed rectifying curve in $\mathbb{H}^{3}(-r)$, lies in the upper half plane $U^{2}$. Then the distance function $\rho=\|\gamma\|$ satisfies $\rho^{2}=\left|a s^{2}+b s+c\right|$ or $\rho^{2}=$ $1+f^{2}(s)$, where $f(s)=c_{1} \sinh \left(\frac{s+s_{0}}{r}\right)+c_{2} \cosh \left(\frac{s+s_{0}}{r}\right)$ and $a, b, c \in \mathbb{R}$.

Proof. Let $\gamma=\gamma(s)$ be a unit speed rectifying curve in $\mathbb{H}^{3}(-r)$. Now, we know that

$$
\gamma(s)=\lambda(s) T_{\gamma}(s)+\mu(s) B_{\gamma}(s),
$$

where $\lambda(s)$ and $\mu(s)$ are differentiable functions.

Now we know that $T_{\gamma}(s)$ and $B_{\gamma}(s)$ are generating a plane, let it be a subset of upper half plane. Therefore $\gamma(s)=(\lambda(s), \mu(s))$ be a curve in $U^{2}$. Then after differentiating the equation (3.12) and using Frenet formulas for $\gamma$, we obtain $\left(1-\lambda^{\prime}\right) T_{\gamma}+\left(\mu \tau_{\gamma}-\lambda \kappa_{\gamma}\right) N_{\gamma}-$ $\mu^{\prime}(s) B_{\gamma}=0$, which implies

$$
\lambda^{\prime}=1, \mu^{\prime}=0, \mu \tau_{\gamma}-\lambda \kappa_{\gamma}=0 .
$$

Therefore $\lambda(s)=s+d_{1}, \mu(s)=d_{2}, \mu(s) \tau_{\gamma}(s)=\lambda(s) \kappa_{\gamma}(s)$. Thus the distance function $\rho^{2}=|g(\gamma, \gamma)|=\left|\frac{\lambda^{2}+\mu^{2}}{\mu^{2}}\right|=\left|\frac{\left(s+d_{1}\right)^{2}+d_{2}^{2}}{d_{2}^{2}}\right|=\left|a s^{2}+b s+c\right|$, where $a=\frac{1}{d_{2}^{2}}, b=\frac{2 d_{1}}{d_{2}^{2}}, c=$ $\frac{d_{1}^{2}+d_{2}^{2}}{d_{2}^{2}}, d_{1}, d_{2} \in \mathbb{R}$. Also from the equation (3.13), we get $\frac{\lambda(s)}{\mu(s)}=\frac{\tau_{\gamma}}{\kappa_{\gamma}}$. Now we know that $\frac{\tau_{\gamma}}{\kappa_{\gamma}}=c_{1} \sinh \left(\frac{s+s_{0}}{r}\right)+c_{2} \cosh \left(\frac{s+s_{0}}{r}\right)=f(s)$, from [7]. Hence $\frac{\lambda}{\mu}=f$. Therefore the distance function, $\rho^{2}=|g(\gamma, \gamma)|=\left|\frac{\lambda^{2}+\mu^{2}}{\mu^{2}}\right|=\left|1+f^{2}\right|$. Thus, $\rho^{2}=1+f^{2}(s)$. This proves the theorem.

Note. Now, we know that $\gamma(s)=\lambda(s) T_{\gamma}(s)+\mu(s) B_{\gamma}(s)$, where $\lambda(s)$ and $\mu(s)$ are differential functions.

(i) Therefore, $g\left(\gamma, T_{\gamma}\right)=\lambda(s)=s+d_{1}$. This is the tangential component of $\gamma(s)$.

(ii) The normal component of $\gamma(s)=\mu(s) B_{\gamma}(s)$. Therefore, $\left\|\gamma^{N}\right\|=d_{2} \neq 0$ i.e., the normal component component of $\gamma(s)$ has a constant length.

(iii) The binormal component of $\gamma(s), g\left(\gamma(s), B_{\gamma}(s)\right)=\mu(s)=d_{2}$, is constant.

Theorem 3.4. Let $\psi(t)$ be a unit speed curve in $\mathbb{R}_{1}^{4}$ and $\gamma$ be a rectifying curve in $\mathbb{H}^{3}(-r)$ with upper half plane as rectifying plane then it has up to a parametrization given by $\gamma(t)=\psi(t) \phi(t)$, or $\gamma(t)=\psi(t) h(t)$.

Proof. Now from Theorem 3.3, we know that $\rho^{2}=a s^{2}+b s+c$ or $\rho^{2}=1+f^{2}(s)$. Let $\rho^{2}=\left|\frac{\left(s+d_{1}\right)^{2}+d_{2}^{2}}{d_{2}^{2}}\right|$, we apply a translation to $s$, such that $\rho^{2}=a s^{2}+1$. Now we define a curve $\psi(t)$ in $\mathbb{R}_{1}^{4}$ by $\psi(s)=\frac{\gamma(s)}{\rho(s)}, \Rightarrow \gamma(s)=\psi(s) \sqrt{a s^{2}+1}$. Then differentiating with respect to $s$, we get

$$
T_{\gamma(s)}=\psi(s) \frac{a s}{\sqrt{a s^{2}+1}}+\psi^{\prime}(s) \sqrt{a s^{2}+1} .
$$

Since, $g(\psi, \psi)=1$, it follows that $g\left(\psi, \psi^{\prime}\right)=0$. Therefore from the equation (3.14), we obtain $1=g\left(T_{\gamma}, T_{\gamma}\right)=g\left(\psi^{\prime}, \psi^{\prime}\right)\left(a s^{2}+1\right)+\frac{a^{2} s^{2}}{a s^{2}+1}$, which implies

$$
g\left(\psi^{\prime}, \psi^{\prime}\right)=\frac{a s^{2}(1-a)+1}{\left(a s^{2}+1\right)^{2}} .
$$


Thus, $\left\|\psi^{\prime}(s)\right\|=\frac{\sqrt{a s^{2}(1-a)+1}}{a s^{2}+1}$. Let $t=\int_{0}^{s}\left\|\psi^{\prime}(u)\right\| d u=\int_{0}^{s} \frac{\sqrt{a s^{2}(1-a)+1}}{a s^{2}+1} d u=\varphi(s)$. Therefore $t=\varphi(s)$ or $s=\varphi^{-1}(t)$. Put this into $\gamma(s)=\psi(s) \sqrt{a s^{2}+1}$, we get $\gamma(t)=$ $\psi(t) \eta\left(\varphi^{-1}(t)\right)=\psi(t) \phi(t)$, where $\eta(s)=\sqrt{a s^{2}+1}, \phi=\eta \circ \varphi^{-1}$. Hence $\gamma(t)=\psi(t) \phi(t)$. Similarly if we take $\rho^{2}=1+f^{2}(s)$ then up to parametrization for $\gamma$ is in the form $\psi(t) h(t)$, which completes the proof.

Theorem 3.5. Let $\gamma=\gamma(s)$ be a unit speed rectifying curve in $\mathbb{H}^{3}(-r)$. Then $T_{\bar{\gamma}}$ can be written in the form, $T_{\bar{\gamma}}=\alpha(s) N_{\gamma}+\beta(s) B \gamma$, where $\alpha(s)=\frac{\lambda \kappa_{\gamma}-\mu \tau_{\gamma}}{a-a \lambda}, \beta(s)=\frac{b-b \lambda+\mu^{\prime}}{a-a \lambda}$ and $a, b \in \mathbb{R}$.

Proof. Let us consider $\gamma=\gamma(s)$ be a unit speed rectifying curve in $\mathbb{H}^{3}(-r)$. Then position vector $\gamma$ of a curve satisfies the equation,

$$
\gamma(s)=\lambda(s) T_{\gamma}(s)+\mu(s) B_{\gamma}(s),
$$

where $\lambda(s)$ and $\mu(s)$ are differentiable functions. On differentiating the equation (3.16), we obtain $T_{\gamma}=\lambda^{\prime} T_{\gamma}+\mu^{\prime}(s) B_{\gamma}+\lambda \kappa_{\gamma} N_{\gamma}-\mu \tau_{\gamma} N_{\gamma}$, which implies

$$
\Rightarrow\left(1-\lambda^{\prime}\right) T_{\gamma}+\left(\mu \tau_{\gamma}-\lambda \kappa_{\gamma}\right) N_{\gamma}-\mu^{\prime}(s) B_{\gamma}=0 .
$$

Since $\gamma=\gamma(s)$ is a unit speed rectifying curve in $\mathbb{H}^{3}(-r)$ therefore $T_{\gamma}=a T_{\bar{\gamma}}-b B_{\gamma}$, where $a, b \in \mathbb{R}$. Thus from the equation (3.17), we get $(a-a \lambda) T_{\bar{\gamma}}+\left(\mu \tau_{\gamma}-\lambda \kappa_{\gamma}\right) N_{\gamma}-(b-b \lambda+$ $\left.\mu^{\prime}\right) B_{\gamma}=0$, which gives

$$
T_{\bar{\gamma}}=\alpha(s) N_{\gamma}+\beta(s) B_{\gamma},
$$

where $\alpha(s)=\frac{\lambda \kappa_{\gamma}-\mu \tau_{\gamma}}{a-a \lambda}$ and $\beta(s)=\frac{b-b \lambda+\mu^{\prime}}{a-a \lambda}, a, b \in \mathbb{R}$. This completes the proof.

Theorem 3.6. Let $\gamma=\gamma(s)$ be a unit speed curve in $\mathbb{H}^{3}(-r)$. Then $\gamma$ is a rectifying general helix if and only if the torsion and curvature of the curve are given by

(i) $\tau_{\gamma}^{2}(s)=\sinh ^{2}\left(\frac{\rho}{r}\right) \cosh ^{2}\left(\frac{s+s_{0}}{r}\right)\left[A \tanh ^{2}\left(\frac{s+s_{0}}{r}\right)+C \tanh \left(\frac{s+s_{0}}{r}\right)+B\right]$,

where $A=\frac{c_{1}^{2} \kappa_{V}^{2} r^{2}}{v^{4}}, B=\frac{c_{2}^{2} \kappa_{V}^{2} r^{2}}{v^{4}}, C=\frac{2 c_{1} c_{2} \kappa_{V}^{2} r^{2}}{v^{4}}$,

(ii) $\kappa_{\gamma}^{2}(s)=\sinh ^{2}\left(\frac{\rho}{r}\right)$, if $A=c_{1}^{2}, B=c_{2}^{2}, C=2 c_{1} c_{2}$.

Proof. By using Theorem 2.3 and Theorem 2.4, we obtain

$$
\tau_{\gamma}^{2}(s)=\frac{\kappa_{V}^{2} r^{2} \sinh ^{2}(\rho / r)}{v^{4}}\left(c_{1} \sinh \left(\frac{s+s_{0}}{r}\right)+c_{2} \cosh \left(\frac{s+s_{0}}{r}\right)\right)^{2},
$$

which implies

$$
\begin{gathered}
\tau_{\gamma}^{2}(s)=A \sinh ^{2}(\rho / r) \sinh ^{2}\left(\frac{s+s_{0}}{r}\right)+C \sinh ^{2}(\rho / r) \sinh \left(\frac{s+s_{0}}{r}\right) \cosh \left(\frac{s+s_{0}}{r}\right) \\
+B \sinh ^{2}(\rho / r) \cosh ^{2}\left(\frac{s+s_{0}}{r}\right)
\end{gathered}
$$

where $A=\frac{c_{1}^{2} \kappa_{V}^{2} r^{2}}{v^{4}}, B=\frac{c_{2}^{2} \kappa_{V}^{2} r^{2}}{v^{4}}, C=\frac{2 c_{1} c_{2} \kappa_{V}^{2} r^{2}}{v^{4}}$. Thus

$$
\begin{gathered}
\tau_{\gamma}^{2}(s)=\sinh ^{2}(\rho / r) \cosh ^{2}\left(\frac{s+s_{0}}{r}\right)\left[A \frac{\sinh ^{2}\left(\frac{s+s_{0}}{r}\right)}{\cosh ^{2}\left(\frac{s+s_{0}}{r}\right)}+C \frac{\sinh \left(\frac{s+s_{0}}{r}\right) \cosh \left(\frac{s+s_{0}}{r}\right)}{\cosh ^{2}\left(\frac{s+s_{0}}{r}\right)}+B\right], \\
\Rightarrow \tau_{\gamma}^{2}(s)=\sinh ^{2}(\rho / r) \cosh ^{2}\left(\frac{s+s_{0}}{r}\right)\left[A \tanh ^{2}\left(\frac{s+s_{0}}{r}\right)+C \tanh \left(\frac{s+s_{0}}{r}\right)+B\right] .
\end{gathered}
$$

Also, again by using Theorem 2.3 and Theorem 2.4, we obtain

$$
\kappa_{\gamma}^{2}(s)=\frac{\tau_{\gamma}^{2}}{\left(c_{1} \sinh \left(\frac{s+s_{0}}{r}\right)+c_{2} \cosh \left(\frac{s+s_{0}}{r}\right)\right)^{2}},
$$




$$
\Rightarrow \kappa_{\gamma}^{2}(s)=\frac{\sinh ^{2}(\rho / r) \cosh ^{2}\left(\frac{s+s_{0}}{r}\right)\left[A \tanh ^{2}\left(\frac{s+s_{0}}{r}\right)+C \tanh \left(\frac{s+s_{0}}{r}\right)+B\right]}{\cosh ^{2}\left(\frac{s+s_{0}}{r}\right)\left[c_{1}^{2} \tanh ^{2}\left(\frac{s+s_{0}}{r}\right)+2 c_{1} c_{2} \tanh \left(\frac{s+s_{0}}{r}\right)+c_{2}^{2}\right]} .
$$

Thus $\kappa_{\gamma}^{2}(s)=\sinh ^{2}(\rho / r)$ if $A=c_{1}^{2}, B=c_{2}^{2}$ and $C=2 c_{1} c_{2}$, which concludes the theorem.

Corollary 3.7. The geodesic curvature $\kappa_{V}$ of rectifying general helix in $H^{3}(-r)$ is given by $\kappa_{V}=\frac{v^{2}}{r}$, where $v$ is the speed of rectifying general helix.

Proof. The proof is obtained from Theorem 3.6.

Theorem 3.8. A curve $\gamma(s)=\exp (\rho(s) V(s))$ in $H^{3}(-r)$ is a rectifying general helix with geodesic curvature $\kappa_{V}(t)=c\left(\cos ^{2}\left(t+t_{0}\right)-a^{2}\right)^{-3 / 2}$ and torsion $\tau(s)=d_{1} \sinh \left(\left(s+s_{0}\right) / r\right)+$ $d_{2} \cosh \left(\left(s+s_{0}\right) / r\right)$ then its curvature $\kappa_{\gamma}$ is of the form $\kappa_{\gamma}=\frac{d_{1}}{c_{1}}$ if and only if

$$
\left|\begin{array}{ll}
c_{1} & c_{2} \\
d_{1} & d_{2}
\end{array}\right|=0
$$

Proof. By using Corollary 9 of [7], we obtain

$$
\begin{gathered}
\kappa_{\gamma}=\frac{d_{1} \sinh \left(\left(s+s_{0}\right) / r\right)+d_{2} \cosh \left(\left(s+s_{0}\right) / r\right)}{c_{1} \sinh \left(\frac{s+s_{0}}{r}\right)+c_{2} \cosh \left(\frac{s+s_{0}}{r}\right)}, \\
\Rightarrow \kappa_{\gamma}=\frac{\left.d_{1}\left(\tanh \left(s+s_{0}\right) / r\right)+A\right)}{c_{1}\left(\tanh \left(\frac{s+s_{0}}{r}\right)+B\right)},
\end{gathered}
$$

where $A=\frac{d_{2}}{d_{1}}$ and $B=\frac{c_{2}}{c_{1}}$.

Thus $\kappa_{\gamma}=\frac{d_{1}}{c_{1}}$ if and only if $A=B$ i.e.

$$
\left|\begin{array}{cc}
c_{1} & c_{2} \\
d_{1} & d_{2}
\end{array}\right|=0
$$

Acknowledgment. We thank to the referees for their valuable suggestions to improve the paper.

\section{References}

[1] P. Alegre, K. Arslan, A. Carriazo, C. Murathan and G. Ozturk, Some special types of developable ruled surface, Hacet. J. Math. Stat. 39 (3), 319-325, 2010.

[2] B. Altunkaya and L. Kula, On spacelike rectifying slant helices in Minkowski 3-space, Turkish J. Math. 42, 1098-1110, 2018.

[3] M. Barros, General helices and a theorem of Lancret, Proc. Amer. Math. Soc. 125 (5), 1503-1509, 1997.

[4] B.Y. Chen, When does the position vector of a space curve always lie in its rectifying plane?, Amer. Math. Monthly 110, 147-152, 2003.

[5] S. Izumiya and N. Takeuchi, New special curves and developable surfaces, Turkish J. Math. 28 (2), 153-163, 2004.

[6] K. Ilarslan, E. Nesovic and M.P. Torgasev, Some characterization of rectifying curves in the Minkowski 3-space, Novi Sad J. Math. 33 (2), 23-32, 2003.

[7] P. Lucas and J.A.O. Yagues, Rectifying curves in the three dimensional hyperbolic space, Mediterr. J. Math. 13, 2199-2214, 2016.

[8] P. Lucas and J.A.O. Yagues, Slant helices in the three dimensional sphere, J. Korean Math. Soc. 54 (4), 1331-1343, 2017. 